\title{
Justificación del postulado de la inmortalidad del alma en la Crítica de la razón práctica de Kant
}

\author{
Justification of the Postulate of the Immortality of \\ the Soul in Kant's Critique of Practical Reason
}

\author{
José Manuel Martínez Blanco \\ Universidade de Santiago de Compostela (España)
}

Recibido: 14.07.2019

Aceptado: 16.09.2019

\section{RESUMEN}

Kant justifica el postulado de la inmortalidad del alma en la Crítica de la razón práctica en tanto que muestra que sin la creencia en la inmortalidad del alma la ley moral perdería su validez. Puesto que si estamos determinados por la ley moral, tenemos la intención moral de promover el bien supremo, la creencia en la posibilidad del bien supremo es una implicación de esta intención, si no aceptamos la posibilidad de un progreso infinito no podemos aceptar la posibilidad del bien supremo y la aceptación de un progreso infinito presupone la aceptación de la inmortalidad del alma.

\section{PALABRAS CLAVE \\ INMORTALIDAD; BIEN SUPREMO; SANTIDAD; PROGRESO \\ INFINITO}

\begin{abstract}
Kant justifies the postulate of the immortality of the soul in his Critique of Practical Reason, inasmuch as he demonstrates that without belief in the immortality of the soul, moral law would lose its validity. Since we are determined by moral law, we have the moral intention to foster the supreme good, the belief in the possibility of the supreme good is an implication of such an intention, and if we do not accept the possibility of an infinite progress, we cannot accept the possibility of
\end{abstract}

Claridades. Revista de filosofía 12/2 (2020), pp. 23-43

ISSN: 1889-6855 ISSN-e: 1989-3787 DL.: PM 1131-2009

Asociación para la promoción de la Filosofía y la Cultura (FICUM) 
the supreme good while the acceptance of an infinite progress presupposes the acceptance of the immortality of the soul.

KEYWORDS

IMMORTALITY; SUPREME GOOD; HOLINESS; INFINITE PROGRESS

\section{INTRODUCCIÓN}

EN LA CRÍTICA DE LA RAZÓN PURA KANT había mostrado lo siguiente: la razón teórica es incapaz de probar la inmortalidad del alma porque no se puede conocer la inmortalidad del alma, tanto la afirmación de la inmortalidad del alma como la negación de la misma sobrepasan los límites de nuestro conocimiento. En la Crítica de la razón práctica Kant dice que, si bien la razón teórica es incapaz de demostrar la inmortalidad del alma, la razón práctica exige a la razón teórica que acepte la inmortalidad del alma. Así pues, en esta obra él llama a la inmortalidad del alma "postulado» en el sentido de exigencia y justifica el postulado de la inmortalidad del alma.

En este artículo básicamente pretendo aclarar la justificación del postulado de la inmortalidad del alma que Kant desarrolla en la «Dialéctica de la razón práctica pura» en la Crítica de la razón práctica y mostrar los problemas de interpretación que esta justificación acarrea. En la «Dialéctica de la razón práctica pura» Kant dedica el apartado «La inmortalidad del alma como postulado de la razón práctica pura» a la justificación del postulado de la inmortalidad del alma en concreto. Ahora bien, para comprender bien la justificación kantiana del postulado de la inmortalidad del alma no debemos restringirnos a analizar el argumento que Kant expone en susodicho apartado, sino que además debemos caracterizar los postulados de la razón práctica pura en general y aclarar la justificación general de los postulados de la razón práctica pura. Por consiguiente, en la primera parte de este artículo caracterizaré los postulados de la razón práctica pura en general y aclararé la justificación general de los mismos. En una segunda parte analizaré detalladamente el argumento a favor del postulado de la inmortalidad del alma que Kant expone en el apartado «La inmortalidad del alma como postulado de la razón práctica pura». 


\section{CaracterizaCión y JUSTIFICACión GENERAL DE LOS POSTUlados DE LA RAZÓN PRÁCTICA PURA ${ }^{\mathrm{I}}$}

Aquí explicaré que cuando Kant habla de los postulados de la razón práctica pura utiliza el concepto "postulado» en el sentido de exigencia. Además mostraré que los postulados de la razón práctica pura, es decir, la libertad de la voluntad, la inmortalidad del alma y la existencia de Dios, según Kant no son frases prácticas, sino frases teóricas que la razón teórica no puede probar. Explicaré que Kant justifica los postulados de la razón práctica en general en tanto que muestra que el estar sometidos a la ley moral nos obliga a aceptar los postulados y que la ley moral sin los postulados no sería válida. Por último aclararé que los postulados de la razón práctica pura son artículos de la fe moral y frases de existencia.

El concepto «postulado» (Postulat) pertenece a las matemáticas. Proviene de la traducción latina del concepto griego «aîtๆ $\mu \alpha$ ». Euclides expuso en su obra Elementos cinco postulados ( $\alpha i \tau \eta ́ \mu \alpha \tau \alpha)$. Él no definió en esta obra los postulados, no obstante está claro que los postulados son para Euclides principios básicos a través de los que pueden ser dadas ciertas construcciones geométricas. Un ejemplo de postulado euclidiano es aquel que exige que un círculo sea descriptible alrededor de un punto cualquiera con un radio cualquiera. Christian Wolf tradujo la palabra latina postulatum con la expresión alemana Heische-Satz. Los postulados son según Wolff frases prácticas que no exigen ninguna prueba y que son derivados a partir de explicaciones. Johann Heinrich Lambert leyó la obra de Euclides Elementos y objetó en contra de Wolff que él había traducido mal la palabra postulatum. Según Lambert los postulados no son Heische-Sätze, sino Forderungen (exigencias). Lambert decía que con la traducción de Wolff se había perdido el carácter activo, práctico de la palabra "postulado». Ade-

\footnotetext{
1 Kant tiene dos concepciones diferentes de razón práctica: él piensa que la razón práctica puede ser una razón práctica empíricamente condicionada o una razón práctica pura. La razón práctica empíricamente condicionada está al servicio de objetivos que proceden de la naturaleza sensible. La razón práctica pura está al servicio de objetivos que proceden de ella misma. La razón práctica pura puede ser también llamada «razón práctica moral». Cf. R. Mosayebi, Das Minimun der reinen praktischen Vernunft. Vom kategorischen Imperativ zum allgemeinen Rechtsprinzip bei Kant, De Gruyter, Berlin y Boston, 2013, pp. 48-49; M. Ivaldo, «Zwei Wege der Kantischen Praktischen Vernunft: Reinhold und Fichte», en G. di Giovanni (ed.), Karl Leonhard Reinhold and the Enlightenment, Springer, Dordrecht, 2010, p. 182.
} 
más objetó contra Wolff que los postulados no son derivados a partir de definiciones y explicaciones. Según Lambert, Wolff entendió mal el método de las matemáticas. ${ }^{2}$

Ahora bien, Kant se refiere a esta polémica en la «Analítica de los principios» de la primera Crítica. Aquí Kant dice que quiere explicar por qué designó los principios de la modalidad con la expresión "postulado». ${ }^{3}$ Él dice que no quiere tomar la palabra "postulado» en el significado «que le han dado algunos autores filosóficos recientes, contra el sentido de los matemáticos, a quienes ella propiamente les pertenece». ${ }^{4}$ Cuando Kant en este pasaje habla del significado matemático de la palabra "postulado» piensa en Lambert y cuando habla de «algunos autores filosóficos recientes» piensa en Wolff y en la escuela wolffiana. ${ }^{5}$ Además él define los postulados de

2 Cf. G. Motta, Die Postulate des empirischen Denkens überhaupt. KrV A 218-236/B 265-287. Ein kritischer Kommentar, De Gruyter, Berlin y Boston, 2012, pp. 71 y $73-$ 77. La palabra Heischesatz es poco conocida incluso por quienes tienen el alemán como lengua materna. Designa una frase en la que se expresa un deseo o una exigencia. Según el diccionario de los hermanos Grimm, a principios del s. XVIII dentro de las matemáticas los conceptos postulatum y Heischesatz eran identificados. Wolff extrapoló la palabra Heischesatz a la filosofía cambiando ligeramente su significado, él utiliza la palabra Heischesatz en el sentido de frase práctica (Übungssatz) que se deriva de una explicación. Supongo que cuando Lambert objeta contra Wolff que los postulados no son Heischesätze sino Forderungen (exigencias) no pretende negar que los postulados sean Heischesätze en el sentido de frases que expresan una exigencia, sino que más bien se refiere a que los postulados no son Heischesätze en el sentido que Wolff le había dado a esta palabra.

3 Cf. KrV A232/B285. Los principios de la modalidad son los postulados del pensamiento empírico, es decir, el postulado de la modalidad, el postulado de la realidad y el postulado de la necesidad. Ellos muestran bajo qué condiciones es admitido un uso empírico de los conceptos modales de la posibilidad, la realidad y la necesidad. Cf. O. Höffe, Immanuel Kant, Beck, München, 2007, pp. 135-136; P. Guyer, «In praktischer Absicht: Kants Begriff der Postulate der reinen praktischen Vernunft», Philosophisches Jahrbuch 104 (1997), p. 9; M. Forschner, «Das Wesen der Erfahrungserkenntnis. Anmerkungen zu Kants 'Grundsätzen des Verstandes'», en N. Fischer (ed.), Kants Grundlegung einer kritischen Metaphysik. Einführung in die «Kritik der reinen Vernunft», Meiner, Hamburg, 2010, pp. 182-183; H. Cornelius, Kommentar zu Kants Kritik der reinen Vernunft, Verl. der Philosophischen Akademie, Erlangen, 1926, pp. 100-102; A. Messer, Kommentar zu Kants Kritik der reinen Vernunft, Strecker und Schröder, Stuttgart, 1922, pp. 113-117; H. Cohen, Kommentar zu Immanuel Kants Kritik der reinen Vernunft, Meiner, Leipzig, 1907, pp. 98-100 y 102-103.

$4 \mathrm{KrV}$ A232-233/B285.

5 Cf. G. Motta, 2012, op. cit., pp. 70 y 281. 
las matemáticas ${ }^{6}$ y dice que la expresión "postulado» no debe estar restringida a su significado matemático sino que esta debe ser también aplicada a los principios de la modalidad «porque ellos no aumentan su concepto de cosas, sino sólo indican la manera como él es, en general, enlazado con la potencia cognoscitiva». ${ }^{7}$ Por otro lado, Kant dice en la segunda Crítica que el significado de los postulados de la razón práctica no debe ser confundido con el significado de los postulados de las matemáticas y que él llamó a las suposiciones de la inmortalidad del alma y de la existencia de Dios "postulados» porque no encontró ninguna palabra mejor. ${ }^{8}$

Como ya dije, Lambert traduce la palabra latina postulatum con el concepto alemán Forderung (exigencia). Cuando Kant habla de los postulados de la razón práctica pura tiene en mente el significado originario de la palabra "postulado». Así pues, según Kant los postulados de la razón práctica pura son exigencias (Forderungen). ${ }^{9}$ Kant no habla en la "Dialéctica de la razón práctica pura» de postulados en el sentido de hipótesis, suposiciones, conjeturas. ${ }^{10}$ Pues si así fuese, entonces estos postulados deberían ser postulados de la razón teórica pura y no postulados de la razón teórica práctica pura, dado que solo la razón teórica puede suponer algo hipotéticamente, conjeturar. Los postulados de la razón práctica pura son exigencias que la razón práctica pura dirige a la razón teórica. ${ }^{11}$ La razón práctica pura exige a la razón teórica aceptar ciertos pensamientos como verdaderos. Pues si la razón teórica no aceptase estos pensamientos, entonces la validez de la ley moral sería socavada. ${ }^{12}$

$6 \mathrm{KrV}$ A234/B287: «Nun heißt ein Postulat in der Mathematik der praktische Satz, der nichts als die Synthesis enthält, wodurch wir einen Gegenstand uns zuerst geben, und dessen Begriff erzeugen, z. B. mit einer gegebenen Linie, aus einem gegebenen Punkt auf einer Ebene einen Zirkel zu beschreiben, und ein dergleichen Satz kann darum nicht bewiesen werden, weil das Verfahren, was er fordert, gerade das ist, wodurch wir den Begriff von einer solchen Figur zuerst erzeugen».

$7 \mathrm{KrV}$ A235-236/B287.

$8 \mathrm{Cf} . \mathrm{KpV}$ A22-23 nota.

9 Cf. G. Motta, 2012, op. cit., p. 84.

10 Cf. L. W. Beck, Kants «Kritik der praktischen Vernunft». Ein Kommentar, Fink, München, 1974, pp. 233-234.

11 Cf. O. Höffe, Kants Kritik der praktischen Vernunft. Eine Philosophie der Freiheit, Beck, München, 2012, p. 183.

12 Cf. G. B. Sala, Kant und die Frage nach Gott. Gottesbeweise und Gottesbeweiskritik in den Schriften Kants, De Gruyter, Berlin, 1990, p. 423; L. W. Beck, 1974, op. 
Según Kant hay tres postulados de la razón práctica pura: la libertad de la voluntad, la inmortalidad del alma y la existencia de Dios. ${ }^{13}$ En la «Dialéctica de la razón práctica pura» de la segunda Crítica Kant caracteriza los postulados de la razón práctica pura como "posiciones teóricas» que están «inseparablemente unidas» con el principio de la autonomía y con la ley moral y «se sustraen a toda posible comprensión de la razón especulativa». ${ }^{14}$ Además en la «Dialéctica de la razón práctica pura» de la segunda Crítica él define el postulado de la razón práctica pura de la siguiente manera: «[...] un postulado de la razón práctica pura (entendiendo yo por tal una proposición teórica, aunque como tal no demostrable, si depende inseparablemente de una ley práctica que vale absolutamente a priori)». ${ }^{15}$ Según esto, los postulados de la razón práctica pura, es decir, la libertad de la voluntad, la inmortalidad del alma y la existencia de Dios, no son frases prácticas, imperativos. ${ }^{16}$ Los postulados de la razón práctica pura son teóricamente indemostrables. La razón especulativa o, dicho de otra manera, la razón teórica pura, no es capaz por sí misma de probar la libertad de la voluntad, la inmortalidad del alma y la existencia de Dios, ella no puede determinar por sus propios medios si estas afirmaciones son verdaderas o falsas. ${ }^{17}$ Los postulados de la razón práctica pura están necesariamente unidos con la ley moral, ellos resultan de la ley moral. ${ }^{18}$

La justificación general de los postulados de la razón práctica pura es la siguiente:

cit., p. 241; F. Ricken, «Die Postulate der reinen praktischen Vernunft (122-148)», en O. Höffe (ed.), Immanuel Kant, Kritik der praktischen Vernunft, Akademie Verlag, Berlin, 2011, p. 189.

13 Cf. L. W. Beck, 1974, op. cit., p. 239; J. Sirovátka, Das Sollen und das Böse in der Philosophie Immanuel Kants. Zum Zusammenhang zwischen kategorischem Imperativ und dem Hang zum Bösen, Meiner,Hamburg, 2015, p. 37.

$14 \mathrm{KpV}$ A216.

$15 \mathrm{KpV}$ A220.

16 Cf. P. Guyer, 1997, op. cit., p. 3; O. Höffe, 2007, op. cit., p. 256; E. Coreth y H. Schöndorf, Philosophie des 17. und 18. Jahrhunderts, Kohlhammer, Stuttgart, 2008, p. 208.

17 Cf. P. Guyer, 1997, op. cit., p. 3; M. Willaschek, «Rationale Postulate. Über Kants These vom Primat der reinen praktischen Vernunft», en H. F. Klemme (ed.), Kant und die Zukunft der europäischen Aufklärung, De Gruyter, Berlin, 2009, p. 261; J. Sirovátka, 2015, op. cit., p. 37.

18 Cf. P. Guyer, 1997, op. cit., p. 3; E. Coreth y H. Schöndorf, 2008, op. cit., p. 208). 
Si nosotros estamos bajo la ley moral, entonces esta provoca en nosotros un "efecto subjetivo", o sea, "la intención conforme con ella [con la ley moral] y necesaria también por ella de promover el supremo bien posible prácticamente». ${ }^{19}$ Pues la ley moral manda promover el bien supremo. ${ }^{20} \mathrm{La}$ ley moral es «apodícticamente cierta por sí misma». ${ }^{21}$ El bien supremo es el objeto de una voluntad determinada por la ley moral. En el bien supremo la felicidad está en proporción con la virtud. ${ }^{22}$ Ahora bien, Kant dice que esta intención "presupone por lo menos que este último [el bien supremo] sea posible». ${ }^{23}$ Esto significa que esta intención implica la creencia en la posibilidad del bien supremo. ${ }^{24}$ Kant ańade que los tres postulados de la razón práctica pura son «las condiciones [...] de la posibilidad del bien supremo». ${ }^{25}$ Así pues, si nosotros no creemos en la libertad de la voluntad, en la inmortalidad del alma y en la existencia de Dios, entonces nosotros no podemos creer tampoco en la posibilidad del bien supremo. ${ }^{26}$ Según Kant, el postulado de la libertad de la voluntad no es solo una condición del bien supremo, sino también una condición de la ley moral. Por el contrario, los postulados de la inmortalidad del alma y de la existencia de Dios son solo condiciones del bien supremo. ${ }^{27}$ Pero los postulados de la inmortalidad del alma y de la existencia de Dios posibilitan también la ley moral, puesto que nosotros no podemos obedecer el mandato de la ley moral que dice que hay que promover el bien supremo si no creemos en la posibilidad del bien supremo y no podemos creer en la posibilidad del bien supremo si no creemos en la inmortalidad del alma y en la existencia de Dios. ${ }^{28}$ Así

$19 \mathrm{KpV}$ A257.

20 Cf. L. W. Beck, 1974, op. cit., pp. 234 y 241; P. Guyer, 1997, op. cit., p. 1997, pp. 2-4; F. Ricken, 2011, op. cit., p. 188.

$21 \mathrm{KpV}$ A257.

22 Cf. KpV A199.

$23 \mathrm{KpV}$ A257.

24 Cf. A. W. Wood, Kant's Moral Religion, Cornell University Press, Ithaca, 1979 p. 151; P. Guyer, 1997, op. cit., p. 7.

$25 \mathrm{KpV}$ A257-258.

26 L. W. Beck, 1974, op. cit., p.241; P. Guyer, 1997, op. cit., p. 2 y 4.

27 Cf. KpV A5-6; véase F. Ricken, 2011, op. cit., p. 189.

28 L. W. Beck, 1974, op. cit., p. 241: «[...] so können wir dem kategorischen Imperativ nur gehorchen und nach dem höchsten Gut streben, wenn wir an die Möglichkeit des höchsten Guts glauben, und dies ist nur möglich, wenn wir an die Existenz dieser Objekte [Unsterblichkeit, Freiheit und Gott] glauben». F. Ricken, 2011, op. cit., p. 
pues, el postulado de la libertad de la voluntad tiene relación directa con la ley moral. Los postulados de la inmortalidad del alma y de la existencia de Dios no tienen relación directa con la ley moral, sino que entran en escena por primera vez a través de otras consideraciones con respecto al concepto del bien supremo. ${ }^{29}$

Según esto, mi estar sometido a la ley moral conlleva la creencia en la libertad de la voluntad, en la inmortalidad del alma y en la existencia de Dios. Si yo hago de la ley moral el motivo de mis acciones, entonces soy empujado hacia los postulados. ${ }^{30}$ La necesidad de aceptar la libertad de la voluntad, la inmortalidad del alma y la existencia de Dios no es un deber, sino una necesidad subjetiva, un requerimiento de la razón (Vernunftbedüfnis). ${ }^{31}$ Kant llama a los tres postulados "presupuestos en un aspecto necesariamente práctico». ${ }^{32}$ Él aquí con la palabra «presupuestos» no quiere decir premisas o hipótesis, sino presupuestos de existencia, esto es, sin estos presupuestos la validez de la ley moral sería destruida, nosotros no seríamos posibles destinatarios de la exigencia de la ley moral. ${ }^{33}$

Los postulados, que, como he mostrado, son deducidos a partir de la ley moral, no son conocimientos. ${ }^{34} \mathrm{El}$ asenso (Führwahrhalten) de los postula-

190: «Wir können der Forderung der reinen praktischen Vernunft, das höchste Gut zu befördern, nur unter der Voraussetzung nachkommen, daß wir das höchste Gut für möglich halten. Die Annahme, daß das höchste Gut möglich ist, und damit die Annahme der Existenz von Freiheit, Unsterblichkeit und Gott ist folglich notwendige Voraussetzung für unser Bemühen, das höchste Gut zu verwirklichen, [...]».

29 Cf. S. Zimmermann, «Kant über 'moralische Argumente'. Worin besteht die Objektivität eines Postulats der reinen praktischen Vernunft?», Archiv für Begriffsgeschichte 58 (2016), p. 96; A. Messer, Kommentar zu Kants ethischen und religions-philosophischen Hauptschriften. Grundlegung zur Metaphysik der Sitten, Kritik der praktischen Vernunft, Religion innerhalb der Grenzen der bloßen Vernunft, Meiner, Leipzig, 1929, pp. 103-104; F. Ricken, 2011, op. cit., p. 189; P. Guyer, 1997, op. cit., p. 8.

30 Cf. S. Zimmermann, 2016, op. cit., pp. 96, 99; O. Höffe, 2007, op. cit., p. 256. Lo cual no significa que no podamos llevar a cabo acciones de acuerdo con la ley moral sin creer en los postulados.

31 Cf. G. B. Sala, 1990, op. cit., p. 422; L. W. Beck, 1974, op. cit., p. 234.

$32 \mathrm{KpV}$ A238.

33 Cf. E. Cilian, Die Ideen in Kants theoretischer und praktischer Philosophie, Lankeit, Königsberg, 1927, p. 49; L. W. Beck, 1974, op. cit., p. 241.

34 Cf. S. Zimmermann, 2016, op. cit., pp. 95; O. Höffe, 2007, op. cit., p. 183; F. Ricken, 2011, op. cit., p. 191; L. W. Beck, 1974, op. cit., p. 241; H. Schwarz, «Ende und Erfüllung. Teleologie und Eschatologie bei Kant», en W. Thiede (ed.), Glauben 
dos no significa saber, sino fe, fe pura de la razón o fe moral..$^{35}$ Según Kant el saber es un asenso suficiente tanto objetiva como subjetivamente. Por el contrario, la fe es un asenso suficiente subjetivamente, pero insuficiente objetivamente. ${ }^{36} \mathrm{El}$ asenso de los postulados es insuficiente objetivamente porque estos no se refieren a un objeto en el espacio y el tiempo que les corresponda, son indemostrables por medio de la razón teórica. Sin embargo, el asenso de los postulados es suficiente subjetivamente porque estos están inseparablemente unidos con la ley moral. ${ }^{37}$

En la Crítica de la razón pura un concepto es objetivamente real si sucede en un juicio de la experiencia y está referido a un objeto. Esta realidad objetiva es la realidad objetiva teórica. Los postulados de la razón práctica pura no tienen realidad objetiva teórica, pero tienen realidad objetiva práctica. ${ }^{38}$ Las ideas transcendentales (el alma, la libertad y Dios) reciben a través de los postulados realidad objetiva en un aspecto práctico. ${ }^{39}$ En los postulados las ideas transcendentales tienen objetos. Pero estos objetos no son objetos de la experiencia posible, no son fenómenos espaciotemporales, sino que son objetos suprasensibles. ${ }^{40}$ Además según Kant este tener objetos «tampoco es todavía conocimiento de estos objetos». ${ }^{41}$ Las ideas no proporcionan conocimiento de estos objetos que tienen porque no se puede tener ninguna intuición de estos objetos. ${ }^{42}$ Kant muestra en la primera Crítica que el conocimiento surge a partir de la aplicación del concepto a la intuición, pero las ideas son conceptos sin intuición que les corresponda.

Según Kant los postulados son frases de existencia. Conforme a lo que Kant dice, en los postulados se postula que el alma, la libertad y Dios

aus eigener Vernunft? Kants Religionsphilosophie und Theologie, Vandenhoeck \& Ruprecht, Göttingen, 2004, pp. 194-195.

35 Cf. F. Ricken, 2011, op. cit., pp. 190-191; L. W. Beck, 1974, op. cit., p. 236; P. Guyer, 1997, op. cit., p. 12; G. B. Sala, 1990, op. cit., p. 422; H. Schwarz, 2004, p. 195.

36 Cf. KrV A822/B850.

37 F. Ricken, 2011, op. cit., p. 191; S. Zimmermann, 2016, op. cit., pp. 99.

38 Cf. S. Zimmermann, 2016, op. cit., pp. 95, 97 y 99.

39 Ibidem, p. 114; T. Kobusch, Selbstwerdung und Personalität. Spätantike Philosophie und ihr Einfluß auf die Moderne, Mohr Siebeck, Tübingen, 2018.

40 Cf. Zimmermann, 2016, op. cit., pp. 98, 100-101 y 114-115.

$41 \mathrm{KpV}$ A243.

42 G. B. Sala, 1990, op. cit., p. 424; F. Ricken, 2011, op. cit., p. 200; L. W. Beck, 1974, op. cit., p. 234; S. Zimmermann, 2016, op. cit., p. 100; P. Guyer, 1997, op. cit., p. 13. 
son reales (wirklich) o existentes (existent, da). Kant utiliza las expresiones Wirklichkeit (realidad), Existenz (existencia) y Dasein (existencia) de manera intercambiable para referirse a las ideas. Kant utiliza los conceptos Wirklichkeit, Existenz y Dasein también en la primera Crítica de manera intercambiable. En la primera Crítica estos son una categoría modal. Esta categoría modal dice que el objeto de mi juicio es dado de hecho a través de la percepción. Pero las ideas en los postulados no son reales en este sentido, sino que la realidad que las ideas reciben en los postulados es una realidad objetiva práctica. ${ }^{43}$

Esta realidad objetiva, estos objetos y esta existencia o realidad, que aquí son asignados a las ideas, son pensados analógicamente. ${ }^{44}$ Según Kant una analogía es una identidad de relación: a y b son diferentes de c y d, pero la relación entre a y b es idéntica a la relación entre c y d. ${ }^{45}$ Por otro lado, nosotros no estamos siempre autorizados a concluir por analogía aquello que legítimamente podemos pensar según una analogía. No se debe confundir el pensar según una analogía (Denken nach einer Analogie) con el concluir por analogía (Schließen nach einer Analogie). Se concluye por analogía si se saca una conclusión debido a una analogía. ${ }^{46}$ La diferencia entre el pensar según una analogía y el concluir por analogía llega a ser clara con ayuda del siguiente ejemplo: se puede pensar que en el caso de los castores el instinto se comporta con respecto a los diques y a las cabańas de los castores como la razón en el caso de los humanos se comporta con respecto a las construcciones humanas. Con ello es pensada una identidad de relación: lo uno es la causa de lo otro, que es el efecto. Si se piensa así, se piensa según una analogía. Pero no estamos legitimados a concluir que, porque el ser humano necesita razón para sus construcciones, los castores tienen razón. Tal conclusión a la que no estamos legitimados a llegar sería una conclusión por analogía. ${ }^{47}$

Ahora bien, según Kant el pensar según una analogía no solo puede ser aplicado al área de lo empírico, sino también al área de lo suprasensible. ${ }^{48}$ Por ejemplo él dice que se puede pensar la causalidad de Dios según una

43 Cf. S. Zimmermann, 2016, op. cit., pp. 103-104.

44 Ibidem, pp. 109 y 112.

45 Cf. KU B448 nota.

46 Cf. S. Zimmermann, 2016, op. cit., pp. 108.

47 Cf. KU B448-449 nota.

48 Cf. S. Zimmermann, 2016, op. cit., p. 110 
analogía. Así pues, se puede pensar legítimamente que la causalidad de Dios está relacionada con los seres vivos organizados igual que el entendimiento humano con las obras de arte. Pero no se está autorizado a sacar la conclusión por analogía de que también Dios tiene entendimiento. ${ }^{49}$ Por consiguiente, los objetos que las ideas tienen en los postulados son representados según una analogía con los objetos espaciotemporales de los conceptos empíricos. Esto no significa que nosotros concluyamos por analogía que, porque los conceptos empíricos tienen objetos en el espacio y el tiempo, las ideas también tienen objetos en el espacio y el tiempo, sino que esto significa que nosotros pensamos que las ideas se comportan con respecto a sus objetos suprasensibles como los conceptos empíricos con respecto a sus objetos empíricos. Con ello nosotros pensamos una identidad de relación: al concepto se contrapone algo. ${ }^{50}$

\section{LA PRUEBA CONCRETA DEL}

\section{POSTULADO DE LA INMORTALIDAD DEL ALMA}

El argumento concreto mediante el cual Kant trata de justificar el postulado de la inmortalidad del alma en la «Dialéctica de la razón práctica pura» en la segunda Crítica es el siguiente: el bien supremo es el objeto de una voluntad pura; la completa adecuación de las intenciones a la ley moral, esto es, la santidad, es una condición necesaria del bien supremo, por tanto es una exigencia necesaria prácticamente; esta no puede ser encontrada en un ser racional finito, sino solo en un avance infinito, el cual es consecuentemente el objeto verdadero de la voluntad; la posibilidad de este avance infinito requiere la inmortalidad del alma; así pues la posibilidad del bien supremo también requiere la inmortalidad del alma. ${ }^{51}$

49 KU B450-451: «Ebenso dürfen wir wohl die Kausalität des Urwesens in Ansehung der Dinge der Welt als Naturzwecke nach der Analogie des Verstandes, als Grundes der Formen gewisser Produkte, die wir Kunstwerke nennen, denken [...] aber wir können daraus, daß unter Weltwesen der Ursache einer Wirkung, die als künstlich beurteilt wird, Verstand beigelegt werden muß, keineswegs nach der Analogie schließen, daß auch dem Wesen, welches von der Natur gänzlich unterschieden ist, in Ansehung der Natur selbst eben dieselbe Kausalität, die wir am Menschen wahrnehmen, zukomme: $[\ldots] \gg$.

50 Cf. S. Zimmermann, 2016, op. cit., pp. 113-114.

51 Cf. KpV A219-220. 
En este apartado quiero aclarar susodicho argumento, el cual se encuentra en el capítulo «La inmortalidad del alma como postulado de la razón práctica pura». Quiero exponer que este argumento acarrea la siguiente dificultad: ¿por qué Kant aduce aquí un argumento que está basado en el concepto de santidad de la voluntad? Pues aquí sería de esperar un argumento distinto. Además quiero explicar que Kant en la segunda frase del argumento dice que la completa adecuación de la intención a la ley moral está en algo que ha sido nombrado en la primera frase de la argumentación, o sea, en el bien supremo, en el objeto necesario, en la ley moral o en la voluntad determinable. La segunda frase del argumento conlleva esta dificultad: ¿dónde está la completa adecuación de la intención a la ley moral? Asimismo quiero aclarar la frase de que la completa conformidad de la intención con la ley moral solo se puede encontrar en un progreso infinito. Esta frase del argumento acarrea la siguiente dificultad: ¿qué razón hay para aceptar este progreso infinito? Por último quiero mostrar que la conclusión de la argumentación es que sin tener fe en la inmortalidad del alma no podríamos aceptar la posibilidad de este progreso que avanza infinitamente y como consecuencia de ello tampoco podríamos aceptar la posibilidad del bien supremo.

Este argumento es un argumento moral. ${ }^{52}$ Con este argumento Kant no intenta probar que la inmortalidad del alma puede ser conocida, sino que intenta justificar la creencia en la inmortalidad del alma. ${ }^{53}$ Así pues, con la ayuda de este argumento intenta justificar que sin la creencia en la inmortalidad del alma no podemos pensar la posibilidad del bien supremo. ${ }^{54}$ Kant piensa que si él lograse demostrar que nosotros sin la creencia en la inmortalidad del alma no nos podemos representar la posibilidad del bien supremo, entonces justificaría la creencia en la inmortalidad del alma. Pues él ya había mostrado que la razón práctica pura debe representarse el bien

52 Cf. L. W. Beck, 1974, op. cit., p. 246. Kant nombra en la Crítica de la razón práctica «prueba[s] moral[es]» o «argumento[s] moral[es]» a aquellas pruebas o argumentos de la filosofía moral que argumentan en pro de los postulados. Cf. S. Zimmermann, 2016, op. cit., p. 92.

53 Cf. E. A. Beach, «The Postulate of Immortality in Kant: To What Extent Is It Culturally Conditioned?», Philosophy East and West 58 (2008), p. 500.

54 Cf. A. W. Wood, 1979, op. cit., pp. 116 y 124. 
supremo «como posible porque ella ordena contribuir con todo lo posible a su realización». 55

Sin embargo, este argumento trae consigo la siguiente dificultad: ¿por qué aduce Kant aquí un argumento aparte que se fundamenta en el concepto de santidad? Pues Kant decía que en el concepto del bien supremo la virtud está necesariamente unida a una felicidad adecuada a ella. ${ }^{56}$ Posteriormente muestra que la unión entre la virtud y una felicidad en proporción con ella no puede ser pensada como analítica, sino que debe ser pensada como sintética, dado que la virtud y la felicidad no son idénticas. ${ }^{57}$ La unión sintética entre la virtud y una felicidad en proporción con ella puede ser pensada como posible solo de la siguiente manera: la virtud es la causa de la felicidad y esta unión entre la virtud y una felicidad en proporción con ella no tiene lugar en el mundo sensible sino en «un mundo inteligible» $^{58}$, o sea, en un Más Allá. Aunque no podamos saber si esta unión en un Más Allá tiene lugar realmente. Ahora bien, Kant podría añadir que tener fe en la inmortalidad del alma es un requisito para que pensemos que en un Más Allá somos partícipes de una felicidad cuya medida sea proporcional a la medida de nuestra virtud. Pero él no ańade esto, sino que desarrolla en el capítulo «La inmortalidad del alma como postulado de la razón práctica pura» un argumento aparte en el que el postulado de la inmortalidad del alma es derivado a partir del concepto de santidad.

Ya he expuesto el argumento concreto a favor de la inmortalidad del alma que Kant desarrolla en la «Dialéctica de la razón práctica pura» en la Crítica de la razón práctica. A continuación analizo en detalle este argumento. La primera premisa del argumento reza: «La obtención del bien supremo en el mundo es el objeto necesario de una voluntad determinable por la ley moral». ${ }^{59}$ En esta premisa se expresa la exigencia de la razón práctica pura de que hay que realizar el bien supremo. Por otro lado, el concepto del mundo que aparece en esta premisa comprende el mundo

$55 \mathrm{KpV}$ A214-115.

56 Cf. KpV A199; véase A. Messer, 1929, op. cit., p. 93; G. B. Sala, 1990, op. cit., p. 401.

57 Cf. KpV A203-204; véase A. Messer, 1929, op. cit., p. 94; G. B. Sala, 1990, op. cit., pp. 406-107.

$58 \mathrm{KpV}$ A207.

$59 \mathrm{KpV}$ A219. «Die Bewirkung des höchsten Guts in der Welt ist das notwendige Objekt eines durchs moralische Gesetz bestimmbaren Willens». 
suprasensible, pues el postulado de la inmortalidad del alma no podría ser derivado a partir de la exigencia de la realización del bien supremo en el mundo sensible. ${ }^{60}$

La segunda premisa es la siguiente: «Pero en ésta, la completa conformidad de las intenciones con ley moral, es la condición suprema del bien supremo». ${ }^{61}$ Kant explica dentro del propio argumento que la completa conformidad de las intenciones con la ley moral es la santidad. ${ }^{62}$ Según Kant una voluntad es santa si es de forma necesaria e inalterable conforme a la ley moral, si concuerda con ley moral y no tiene motivos alternativos que la determinen. ${ }^{63}$ Así pues, una voluntad santa es "una voluntad incapaz de máxima alguna contraria a la ley moral». ${ }^{64}$ Un ser racional finito no puede tener una voluntad santa porque este tiene junto con la ley moral aún otros motivos que determinan su voluntad, esto es, móviles sensibles y necesidades. ${ }^{65}$ Por otro lado, Kant contrapone la santidad a la virtud. Él dice que el estado moral en el que el ser humano puede estar «es la virtud, es decir, la intención moral en la lucha, y no la santidad en la presunta posesión de una completa pureza de intenciones de la voluntad». ${ }^{66}$ Esto significa que nuestra intención moral debe imponerse y demostrar su fuerza luchando contra nuestras inclinaciones y pasiones.

Ahora bien, la segunda premisa lleva aparejada una dificultad: Kant se refiere en la segunda premisa con las palabras in diesem $^{67}$ a algo en lo que está la completa conformidad de las intenciones con ley moral. ¿A qué

60 Cf. F. Ricken, 2011, op. cit., pp. 197-198.

$61 \mathrm{KpV}$ A219. «In diesem aber ist die völlige Angemessenheit der Gesinnungen zum moralischen Gesetze die oberste Bedingung des höchsten Guts».

62 Cf. KpV A220.

63 Cf. M. Willaschek, 2009, op. cit., p. 262; G. B. Sala, Kants «Kritik der praktischen Vernunft». Ein Kommentar, Wissenschaftliche Buchgesellschaft, Darmstadt, 2004, p. 276 nota 280.

$64 \mathrm{KpV}$ A57.

65 Cf. KpV A57, 149.

$66 \mathrm{KpV}$ A151.

67 Como acabamos de ver, José Rovira Armengol en su traducción de la Crítica de la razón práctica elige las palabras «en ésta» para traducir al español las palabras alemanas in diesem. Pero es importante señalar que la palabra diesem no es un pronombre demostrativo femenino, sino un pronombre demostrativo masculino o neutro. Por lo tanto, las palabras in diesem solo pueden referirse a un sustantivo que en alemán sea masculino o neutro, esto es, al bien supremo, al objeto necesario, a la ley moral o a la voluntad determinable. 
se refiere Kant en la segunda premisa con las palabras in diesem? ¿Dónde está la completa conformidad de las intenciones con ley moral? Se podría interpretar que Kant en la segunda premisa con las palabras in diesem se refiere al bien supremo. Así pues, se podría interpretar que Kant en la segunda frase quiere decir que en el concepto del bien supremo la completa adecuación de las intenciones a la ley moral es la condición suprema del bien supremo. Esto significaría que la santidad está en el concepto del bien supremo, que ella es la condición suprema del bien supremo. Pero Kant había mostrado que la condición suprema (oberste Bedingung) del bien supremo es la virtud. ${ }^{68}$ Como ya expliqué, Kant decía que la virtud y la felicidad están necesariamente unidas en el concepto del bien supremo y que la relación entre la virtud y la felicidad es una relación proporcionada. Él decía que en el concepto del bien supremo la felicidad está «distribuida en proporción exacta a la moralidad». ${ }^{69}$ Esto significa que a un grado de virtud le corresponde un grado de felicidad, que la virtud puede tener diferentes grados. Pero la completa conformidad de las intenciones con la ley moral o la santidad no puede significar diferentes grados de virtud. Así pues, si Kant con la segunda premisa quisiese decir que la completa conformidad de las intenciones con la ley moral está en el concepto del bien supremo, entonces esta premisa sería difícilmente reconciliable con lo que Kant antes había dicho sobre el bien supremo. ${ }^{70}$

Otra posible interpretación sería que Kant en la segunda premisa con las palabras in diesem no se refiere al bien supremo sino a la ley moral. Así pues, se podría interpretar que Kant en la segunda premisa quiere decir que en el concepto de la ley moral es pensada la completa conformidad de las intenciones con la ley moral, que la completa adecuación de las intenciones a la ley moral no está en el concepto del bien supremo, sino en el concepto de la ley moral. Pero la completa conformidad de las intenciones con la ley moral o santidad no puede ser una característica conceptual de la ley moral. Pues Kant dice que tanto los seres humanos como Dios están sometidos a leyes morales. ${ }^{71}$ No obstante, según Kant, Dios es santo ${ }^{72}$ y

68 Cf. L. W. Beck, 1974, op. cit., p. 247.

$69 \mathrm{KpV}$ A199.

70 Cf. G. B. Sala, 2004, op. cit., p. 277; L. W. Beck, 1974, op. cit., p. 247.

$71 \mathrm{Cf} . \mathrm{KpV}$ A57.

72 GMS AB39; véase P.-B. Park, Das höchste Gut in Kants kritischer Philosophie. Eine Untersuchung über den Zusammenhang von kritischer Ethik und Metaphysik, 
los seres humanos, como ya dije, no son santos. Si la santidad fuera una característica conceptual de la ley moral los seres humanos también serían santos. Una tercera interpretación sería que Kant en la segunda premisa con las palabras in diesem se refiere a la voluntad determinable, que la completa conformidad de las intenciones con la ley moral está en la voluntad determinable. Según esto se podría interpretar que Kant con la segunda premisa quiere decir que la ley moral exige de nosotros completa conformidad de las intenciones con sus órdenes. Pero esta interpretación sería complicada y Kant normalmente no se expresa así. Por consiguiente, queda abierta la cuestión de cómo debe ser interpretada la segunda premisa de la argumentación.

Luego Kant indica en su argumento que la santidad es «una perfección que no puede alcanzar ningún ente racional del mundo sensible en ningún momento de su existencia». ${ }^{73}$ Esto significa que la voluntad de los seres raciones en esta vida tiene motivos opuestos que la determinan. ${ }^{74}$ Acto seguido Kant dice: «[A] Mas como, no obstante, se exige [la completa conformidad de las intenciones con la ley moral] necesaria prácticamente, [B] sólo puede hallarse en un progreso proseguido hasta el infinito esa perfecta conformidad, [...]». ${ }^{75}$ Con la frase A Kant expresa la exigencia de la santidad. Nosotros no sabemos por qué esta es exigida, pues, como ya fue señalado, no está claro cómo debe ser interpretada la segunda premisa del argumento. Por otro lado, con la frase B Kant no quiere decir que la santidad es una parte de un avance infinito, de una serie infinita. Más bien él quiere decir que Dios ve este avance infinito y la santidad como equivalentes, este avance sin fin cuenta en la intuición intelectual de Dios como santidad. ${ }^{76}$

En la frase B cuando Kant habla de un progreso infinito parece querer decir que nosotros nos acercamos a la santidad no solo en esta vida, sino también más allá de la vida terrenal. ${ }^{77}$ Yo me quiero referir aquí al escrito El fin de todas las cosas para aclarar el concepto de progreso infinito. En

tesis doctoral, Köln, 2000, pp. 108-109; G. B. Sala, op. cit., 2004, p. 276 nota 280.

$73 \mathrm{KpV}$ A220.

74 Cf. E. A. Beach, 2008, op. cit., p. 500.

$75 \mathrm{KpV}$ A220.

76 Cf. A. W. Wood, 1979, op. cit., pp. 119-121; L. W. Beck, 1974, op. cit., p. 248; G.

B. Sala, 2004, op. cit., p. 279.

77 Cf. A. W. Wood, 1979, op. cit., p. 123. 
este escrito Kant no intenta refutar la escatología, sino que intenta llevar a cabo una apropiación filosófica de escatología. ${ }^{78}$ En este marco él trata la duración infinita o eternidad. Al inicio del escrito aclara la expresión «él va del tiempo a la eternidad» (er geht aus der Zeit in die Ewigkeit), una expresión que según Kant era corriente y se emitía con vistas a un moribundo. Kant dice que en esta frase uno no puede referirse con la palabra «eternidad» a un tiempo. Pues si en la expresión «él va del tiempo a la eternidad» uno se refiriese con la palabra «eternidad» a un tiempo, entonces se estaría diciendo con esta expresión que él va del tiempo al tiempo, con lo que esta expresión sería banal. ${ }^{79}$ Kant dice que en esta expresión con la palabra «eternidad» uno tiene que referirse a «un fin de todos los tiempos, perdurando el hombre sin cesar». ${ }^{80}$ Él añade que este avance ininterrumpido del ser humano es «una magnitud inconmensurable con el tiempo (duratio noumenon), de la que ningún concepto podemos formarnos (fuera del negativo)». ${ }^{81}$ Además Kant señala en otro lugar del escrito que el concepto de duración infinita o de duración como eternidad es «un concepto negativo de la duración eterna, [...] con el cual no avanzamos ni un solo paso en nuestro conocimiento». ${ }^{82}$ Del escrito El fin de todas las cosas se puede sacar la conclusión de que Kant no considera el progreso infinito del que habla en la frase B como un progreso temporal. Además se puede confirmar que según Kant no se puede conocer este avance infinito. ${ }^{83}$

Ahora bien, la frase B nos coloca ante el siguiente problema: ¿por qué se debe aceptar un acercamiento infinito a la santidad que va más allá de la vida terrenal? ${ }^{84} \mathrm{O}$ sea, Kant decía en su argumentación que la santidad es exigida y que nosotros no la podemos alcanzar en el mundo sensible. Sería de esperar que Kant a continuación argumentase a favor de la necesidad

78 Cf. H. A. Salmony, Kants Schrift Das Ende aller Dinge, EVZ, Zürich, 1962, p. 19; J. Wohlmuth, Mysterium der Verwandlung. Eine Eschatologie aus katholischer Perspektive im Gespräch mit jüdischem Denken der Gegenwart, Schöningh, Paderborn, 2005, p. 105.

79 Cf. EaD A595; véase H. A. Salmony, 1962, op. cit., p. 15; J. Wohlmuth, 2005, op. cit., p. 104

80 EaD A495.

81 EaD A494-495; véase G. B. Sala, 1990, op. cit., pp. 277-278; H. A. Salmony, 1962, op. cit., p. 15.

82 EaD A510; véase F. Ricken, 2011, op. cit., p. 199.

83 Cf. A. W. Wood, 1979, op. cit., pp. 122-124; E. A. Beach, 2008, op. cit., p. 503.

84 Cf. E. A. Beach, 2008, op. cit., p. 501. 
de la aceptación de que podemos alcanzar la santidad en un mundo suprasensible. ${ }^{85}$ Pero él no hace esto sino que habla de un avance infinito que va más allá de la muerte. ¿Por qué habla de un progreso infinito? Una posible respuesta a esta pregunta sería que la aceptación de que podemos alcanzar la santidad en la otra vida según la opinión de Kant es peligrosa para la moral. ${ }^{86}$ Pues si pensásemos que podemos alcanzar la santidad estaríamos en la siguiente situación: o bien debilitaríamos la exigencia moral, o bien tensaríamos en exceso nuestra capacidad y caeríamos «en exaltados sueños teosóficos, totalmente contradictorios con el conocimiento de nosotros mismos». ${ }^{87} \mathrm{La}$ creencia de que solo nos podemos acercar a la santidad en un progreso infinito nos protegería ante este peligro. ${ }^{88}$

Luego Kant dice: «Pero este progreso infinito sólo es posible suponiendo una existencia que perdure hasta el infinito y una personalidad del mismo ente racional (lo que se denomina inmortalidad del alma)». ${ }^{89}$ Esto significa que solo podemos aceptar la posibilidad de un acercamiento infinito a la santidad si creemos en la inmortalidad del alma. ${ }^{90}$ De esta manera él justifica que no nos podemos representar la posibilidad del bien supremo si no creemos en la inmortalidad del alma.

\section{REFERENCIAS BIBLIOGRÁFICAS:}

Beach, E. A.: "The Postulate of Immortality in Kant: To What Extent Is It Culturally Conditioned?» en Philosophy East and West 58, 2008, pp. 492-523.

Beck, L. W.: Kants «Kritik der praktischen Vernunft». Ein Kommentar. München: Fink, 1974.

Cohen, H.: Kommentar zu Immanuel Kants Kritik der reinen Vernunft. Leipzig: Meiner, 1907.

85 Cf. A. W. Wood, 1979, op. cit., p. 117.

86 Cf. E. A. Beach, 2008, op. cit., pp. 505-506; A. W. Wood, 1979, op. cit., pp. $117-$ 118.

$87 \mathrm{KpV} 221$.

88 Cf. E. A. Beach, 2008, op. cit., p. 506; A. Messer, 1929, op. cit., p. 99; G. B. Sala, 2004, op. cit., p. 279; F. Ricken, 2011, op. cit., p. 199.

$89 \mathrm{KpV}$ A220.

90 Cf. A. W. Wood, 1979, op. cit., p. 121. 
Coreth, E. y Schöndorf, H.: Philosophie des 17. und 18. Jahrhunderts. Stuttgart: Kohlhammer, 2008.

Cornelius, H.: Kommentar zu Kants Kritik der reinen Vernunft. Erlangen: Verl. der Philosophischen Akademie, 1926.

Forschner, M.: «Das Wesen der Erfahrungserkenntnis. Anmerkungen zu Kants 'Grundsätzen des Verstandes'» en Fischer N.: Kants Grundlegung einer kritischen Metaphysik. Einführung in die "Kritik der reinen Vernunft». Hamburg: Meiner, 2010, pp. 167-184.

Guyer, P.: «In praktischer Absicht: Kants Begriff der Postulate der reinen praktischen Vernunft» en Philosophisches Jahrbuch 104, 1997, pp. 1-18.

Höffe, O.: Kants Kritik der praktischen Vernunft. Eine Philosophie der Freiheit. München: Beck, 2012.

Höffe, O.: Immanuel Kant. München: Beck, 2007.

Ivaldo, M.: «Zwei Wege der Kantischen Praktischen Vernunft: Reinhold und Fichte» en Di Giovanni, G.: Karl Leonhard Reinhold and the Enlightenment. Dordrecht: Springer, 2010, pp. 181-193.

Kant, I.: Kritik der praktischen Vernunft \& Grundlegung zur Metaphysik der Sitten. Frankfurt: Suhrkamp, 2014. Traducción: Kant, I.: Critica de la razón práctica (traducción de José Rovira Armengol). Buenos Aires: Losada, 1973.

Kant, I.: Kritik der reinen Vernunft 1-2. Frankfurt: Suhrkamp, 2014. Traducción: Kant, I.: Crítica de la razón pura (traducción de Mario Caimi). Buenos Aires: Colihue, 2007.

Kant, I.: Kritik der Urteilskraft. Beilage: Erste Einteilung in die Kritik der Urteilskraft, Hamburg: Meiner, 2009. Traducción: Kant I., Crítica del Juicio (traducción de Manuel García Morente). Madrid: Tecnos, 2007.

Kant I.: Schriften zur Anthropologie, Geschichtsphilosophie, Politik und Pädagogik 1. Frankfurt: Suhrkamp, 2014. Traducción: Kant, I.: Filosofia de la historia (traducción de Eugenio Imaz). México: El Colegio de México, 1941.

Kobusch, T.: Selbstwerdung und Personalität. Spätantike Philosophie und ihr Einfluß auf die Moderne, Tübingen, Mohr Siebeck, 2018.

Messer, A.: Kommentar zu Kants ethischen und religions-philosophischen Hauptschriften. Grundlegung zur Metaphysik der Sitten, Kritik der praktischen Vernunft, Religion innerhalb der Grenzen der bloßen Vernunft. Leipzig: Meiner, 1929. 
Messer, A.: Kommentar zu Kants Kritik der reinen Vernunft. Stuttgart: Strecker und Schröder, 1922.

Mosayebi, R.: Das Minimun der reinen praktischen Vernunft. Vom kategorischen Imperativ zum allgemeinen Rechtsprinzip bei Kant. Berlin y Boston: De Gruyter, 2013.

Motta, G.: Die Postulate des empirischen Denkens überhaupt. KrV A 218236/B 265-287. Ein kritischer Kommentar. Berlin y Boston: De Gruyter, 2012.

Park, P.-B.: Das höchste Gut in Kants kritischer Philosophie. Eine Untersuchung über den Zusammenhang von kritischer Ethik und Metaphysik, tesis doctoral, Köln, 2000.

Ricken, F.: «Die Postulate der reinen praktischen Vernunft (122-148)» en: Höffe O.: Immanuel Kant, Kritik der praktischen Vernunft. Berlin: Akademie Verlag, 2011, pp. 187-202.

Sala, G. B.: Kants "Kritik der praktischen Vernunft». Ein Kommentar. Darmstadt: Wissenschaftliche Buchgesellschaft, 2004.

Sala, G. B.: Kant und die Frage nach Gott. Gottesbeweise und Gottesbeweiskritik in den Schriften Kants. Berlin: De Gruyter, 1990.

Salmony, H. A.: Kants Schrift Das Ende aller Dinge. Zürich: EVZ, 1962.

Schwarz, H.: «Ende und Erfüllung. Teleologie und Eschatologie bei Kant» en Thiede W.: Glauben aus eigener Vernunft? Kants Religionsphilosophie und Theologie. Göttingen: Vandenhoeck \& Ruprecht, 2004, pp. 191206.

Sirovátka, J.: Das Sollen und das Böse in der Philosophie Immanuel Kants. Zum Zusammenhang zwischen kategorischem Imperativ und dem Hang zum Bösen. Hamburg: Meiner, 2015.

Willaschek, M.: «Rationale Postulate. Über Kants These vom Primat der reinen praktischen Vernunft» en Klemme H. F.: Kant und die Zukunft der europäischen Aufklärung, Berlin: De Gruyter, 2009, pp. 251-268.

Wohlmuth, J.: Mysterium der Verwandlung. Eine Eschatologie aus katholischer Perspektive im Gespräch mit jüdischem Denken der Gegenwart. Paderborn: Schöningh, 2005.

Wood, A. W.: Kant's Moral Religion. Ithaca: Cornell University Press, 1970 .

Zilian, E.: Die Ideen in Kants theoretischer und praktischer Philosophie, Königsberg: Lankeit, 1927. 
Zimmermann, S.: «Kant über 'moralische Argumente'. Worin besteht die Objektivität eines Postulats der reinen praktischen Vernunft?» en Archiv für Begriffsgeschichte 58, 2016, pp. 91-117.

José Manuel Martínez Blanco es licenciado en filosofía por la Universidade de Santiago de Compostela y ha cursado un máster en filosofía en la Universidad de Bonn.

Lineas de investigación:

Kant, idealismo alemán

Dirección electrónica: josemanuel1859@gmail.com 
